\title{
Ischemia Modified Albumin Can Predict Necrosis at Incarcerated Hernias
}

\author{
Hüseyin Kadioğlu, ${ }^{1}$ Dilek Ömür, ${ }^{2}$ Süleyman Bozkurt, ${ }^{1}$ Ekrem Ferlengez, ${ }^{3}$ Naim Memmı, ${ }^{1}$ \\ Yeliz Emine Ersoy, ${ }^{1}$ Gökhan Çıpe, ${ }^{1}$ and Mahmut Müslümanoğlu ${ }^{2}$ \\ ${ }^{1}$ General Surgery Department, Medical Faculty, Bezmialem Vakıf University Hospital, Adnan Menderes Bulvarı Vatan Caddesi, \\ 34093 Fatih, İstanbul, Turkey \\ ${ }^{2}$ Anesthesiology Department, Medical Faculty, Dokuz Eylül University, Izmir, Turkey \\ ${ }^{3}$ General Surgery Department, Haseki Training and Research Hospital, Istanbul, Turkey
}

Correspondence should be addressed to Hüseyin Kadioğlu; huseyinkadioglu@gmail.com

Received 6 August 2013; Revised 25 October 2013; Accepted 7 November 2013

Academic Editor: Valeria Barresi

Copyright (C) 2013 Hüseyin Kadioğlu et al. This is an open access article distributed under the Creative Commons Attribution License, which permits unrestricted use, distribution, and reproduction in any medium, provided the original work is properly cited.

\begin{abstract}
Purpose. To evaluate the predictive effect of IMA in incarcerated hernias. Methods. Three groups $(n=7)$ of rats were operated. Group I aimed to mimic incarceration, group II aimed the strangulation, and group III was the sham group. IMA and LDH measurements were made. Results. IMA levels were significantly higher in strangulation mimicking group and IMA levels were normal at postoperative 6th hour in incarceration mimicking group. LDH levels were significantly higher in both incarceration and strangulation mimicking groups. Conclusion. IMA seems to be an effective marker in incarcerated hernias to predict necrosis. But we need further studies to generalise this hypothesis.
\end{abstract}

\section{Introduction}

Among all emergency cases the ratio of incarcerated/strangulated hernias differs across the countries. But it is well known that hernia is the common cause of small bowel obstruction, and mortality and morbidity rates increase in case of strangulation. During acute ischemic conditions, metal binding capacity of albumin is modified and a metabolic variant with reduced transition metal binding occurs. This change is quantifiable and commonly known as ischemia modified albumin (IMA). Recently, IMA measurement has been proposed as a sensitive marker for the diagnosis of myocardial ischemia presenting with typical acute chest pain [1]. However, IMA levels are also known to increase in other ischemic conditions, as well as to be an indicator of oxidative stress, and may not be specific for cardiac ischemia. Recently, Turedi et al. [2] showed that the level of IMA may be of use in the diagnosis of pulmonary embolism, another ischemic condition. Incarceration is the state of an external hernia which cannot be reduced into the abdomen. Implying an increased risk of obstruction and strangulation, incarceration has great importance [3]. Incarcerated external hernias are the second most common cause of small intestinal obstructions [4]. Roughly $5 \%$ of all patients having an operation for external hernia are explored as an emergency. Strangulated external hernia is a relatively common and serious surgical emergency and accounts for most of the deaths from this condition [5]. Clinical validation of any test for ischemia is difficult as there is no accepted diagnostic gold standard. And the preoperative diagnosis or prediction of ischemia is important for surgeons because ischemia changes the operative strategy. Thus we planned this study and aimed to show relation between the intestinal ischemia and IMA.

1.1. Materials and Methods. Our study was initiated after Çanakkale Onsekiz Mart University (COMU) Animal Ethics Committee approval (approval number 2010/11.02) and took place in COMU laboratories. Twenty Wistar albino rats were used weighing 153-207 g and were housed in optimum conditions. In this study rats were divided into sham group, strangulation group, and incarceration group. Each group 
included 7 rats. Before operation, they were kept for two weeks in cages with five animals each and 12 : 12-hour artificial light to dark cycles. The rats received standard diet and water. We aimed to mimic incarceration by short clamping (45 minutes) and strangulation by 6 hours clamping.

1.2. Operative Technique. Ketamine $\mathrm{HCl}$ (Ketalar, Pfizer, Turkey) $35 \mathrm{mg} / \mathrm{kg}$ and xylazine (Basilazin Flacon \%2, Turkey) $5 \mathrm{mg} / \mathrm{kg}$ were administered intraperitoneally as anesthetic agents.

Group I (incarceration group, $n=7$ ). After midline laparotomy, mesenteric vascular structures of small intestine were clamped by a vascular clamp. Clamping was terminated after 45 minutes and reperfusion of small intestine was observed. Reperfusion was confirmed with the restoration of color. Then blood samples were taken [for IMA (Imal) and lactate dehydrogenase (LDH)] and midline incision was closed separately by polypropylene sutures. Blood samples were taken again at postoperative 6th hour (Ima2).

Group II (strangulation group, $n=7$ ). After midline laparotomy, mesenteric vascular structures of small intestine were ligated by $2 / 0$ polypropylene sutures and midline incision was closed separately by polypropylene sutures. Midline laparotomy was done again at 6th hour, intestinal necrosis were seen, and blood samples were taken [for IMA (Ima2) and $\mathrm{LDH}$. Rats were sacrificed by high dose anesthetic agents.

Group III (sham group, $n=7$ ). After midline laparotomy then blood samples were taken [for IMA (Ima1) and LDH] and midline incision was closed separately by polypropylene sutures. Blood samples were taken again at postoperative 6 th hour (Ima2).

There is no standard rat method to simulate incarceration. But incarceration is a known ischemia reperfusion process and total vascular clamping is a widely used method to investigate ischemia reperfusion in rats. So we used this method to simulate incarceration in group I. Also total occlusion of all vascular support of the small intestine is used in group II to provide the smaller amounts of ischemic/necrotic tissue in group I and group II and to prevent the potential effect of ischemic/necrotic tissue amount on IMA levels.

1.3. IMA Measurement. Reduced cobalt to albumin binding capacity (IMA levels) was analyzed using the rapid and colorimetric method developed by Bar et al. [6]. The results were reported as absorbance unit (ABSU). Results higher than 0,4 ABSU were estimated as ischemia and lower than 0,4 ABSU were estimated as normal.

1.4. Statistical Analysis. Quantitative variables were compared by nonparametric tests. Kruskal-Wallis test was used to compare the groups and probability $(P)$ values less than 0.05 were considered significant. The significance of the parameters in Kruskal-Wallis test was confirmed by MannWhitney $U$ test and $P$ values less than 0,016 were considered significant.
TABLE 1: Comparison of groups for Imaio and LDH levels.

\begin{tabular}{lcc}
\hline & IMA1 & LDH \\
\hline Group I versus & Group I $<$ group II & Group I $=$ group II \\
group II & $P: 0,004$ & $P: 0,443$ \\
\hline Group I versus & Group I $>$ group III & Group I $>$ group III \\
group III & $P: 0,001$ & $P: 0,001$ \\
\hline Group II versus & Group II $>$ group III & Group II $>$ group III \\
group III & $P: 0,002$ & $P: 0,002$ \\
\hline
\end{tabular}

Kruskal-Wallis test is the standard variance analysis test to compare the means of 3 or more groups. Kruskal-Wallis test gives us the difference between the groups, but it does not give any idea about which group is the cause of this difference. We used Mann-Whitney $U$ test with Bonferroni correction (the $P$ value for Mann-Whitney $U$ test by Bonferroni correction is found to be $0,05 /$ number of groups $=0,016$ for this study).

\section{Results}

Mean IMA1 levels of group I, group II, and group III were $0,41 \pm 0,01$ (range 0,40-0,45), 0,46 $\pm 0,16$ (range 0,44-0,48), and 0,34 $\pm 0,03$ (range 0,28-0,39), respectively $(P<0,001)$.

Mean LDH levels were 819,71 \pm 34,53 (range 766-874), $808,57 \pm 24,69$ (range 774-841), and 514,14 \pm 19,92 (range 487-545) for groups I, II, and III, respectively. Comparison of groups for Imal and LDH levels was given in Table 1.

The difference of IMA levels between group I and group II was not significant at 6th hour (IMA2) $(P: 0,71)$.

Biochemical results of groups were given in Table 2.

\section{Discussion}

During acute myocardial ischemia, the ability of binding ions such as copper, zinc, and cobalt is decreased; therefore a form of albumin is produced, which is described as ischemia modified albumin (IMA). The IMA was registered by the United States Food and Drug Administration as a marker of myocardial ischemia. The test is based on the decreased ability of albumin to bind cobalt due to the structural change of $\mathrm{NH}_{2}$ which develops in the ischemic environment. It is important to emphasise that the decreased ability of albumins to bind cobalt occurs in hypoxia, acidosis, sodium and calcium pump malfunctions, and tissue damage caused by free radicals $[7,8]$. It has been shown that the concentration of IMA increases within a couple of minutes from the onset of ischemia, remains elevated until 6 to 12 hours, and returns to its normal range after 24 hours [9].

But recent studies showed that IMA increases in mesenteric ischemia, stroke, and cerebrovascular accidents as well $[1,10]$. Gunduz et al. [11] found significantly higher IMA levels in superior mesenteric artery thromboembolism and they concluded that IMA can be used in the diagnose of mesenteric ischemia [12].

The emergency presentation of hernias is a common feature of the general surgical workload. Incarcerated and/or strangulated hernias usually require emergency surgical 
TABLE 2: Biochemical results of groups.

\begin{tabular}{lccc}
\hline & $\begin{array}{c}\text { Ima1 } \\
(\mathrm{ABSU})\end{array}$ & $\begin{array}{c}\text { Ima2 } \\
(\mathrm{ABSU})\end{array}$ & $\begin{array}{c}\text { LDH levels } \\
(\mathrm{UI} / \mathrm{L})\end{array}$ \\
\hline & 0,41 & 0,37 & 827,00 \\
Group I & 0,45 & 0,34 & 766,00 \\
(ischemia mimicking group) & 0,42 & 0,36 & 874,00 \\
& 0,41 & 0,37 & 841,00 \\
& 0,40 & 0,33 & 828,00 \\
& 0,42 & 0,35 & 796,00 \\
Group II (necrosis group) & 0,41 & 0,30 & 806,00 \\
\hline & & 0,48 & 826,00 \\
& & 0,47 & 774,00 \\
& & 0,44 & 841,00 \\
& & 0,46 & 823,00 \\
& & 0,46 & 779,00 \\
& & 0,44 & 805,00 \\
& & 0,48 & 812,00 \\
\hline & 0,34 & 0,36 & 487,00 \\
& 0,39 & 0,35 & 499,00 \\
& 0,33 & 0,37 & 527,00 \\
& 0,35 & 0,33 & 545,00 \\
& 0,34 & 0,33 & 503,00 \\
& 0,35 & 511,00 \\
& & 0,30 & 527,00 \\
\hline
\end{tabular}

intervention. Patients requiring emergency repair tend to be a member of an elderly and unfit population [13-15].

It is known that most of incarcerated hernias are groin hernias. The rate of incarcerated groin hernias changes by countries. Ge et al. found this rate $12,8 \%$ in China [16], Nilsson et al. found 6\% [17], and Bickell et al. found 13\% [18]. Ge et al. concluded that the main causes of high incarceration rates are economic problems and lack of insurance [16].

Morbidity and mortality risks of incarcerated hernias are increased by longer duration of symptoms [13-15, 19,20], type of hernia (femoral rather than inguinal hernia) [17], a high American Society of Anesthesiology (ASA) score [14, 17, 19, $21]$, the presence of comorbidities [14, 15], and the presence of bowel necrosis $[14,17,19]$. This study aimed to predict necrosis by IMA levels and explore the impact of LDH.

We can evaluate ASA score, duration of symptoms, and the presence of comorbidities preoperatively, but the need for an intestinal resection is unclear until surgery. This has been reported to be the case even for experienced surgeons where the sensitivity has been reported to be, at best, $48 \%$ [22].

Maeda et al. reported that the serum creatine phosphokinase (CPK) level was a useful marker for the diagnosis of a strangulated hernia [23], and Ohira et al. found that CPK, lactate dehydrogenase, and metabolic acidosis, in addition to ultrasound and enhanced computed tomography, were of value in diagnosing strangulation of the intestine in some cases [24]. A definitive diagnosis of strangulation of the intestine, however, can only be made during a surgical exploration $[5,25]$. The current series showed a significant increase in the need for a resection of the small intestine when the time from onset of symptoms to surgery was more than 12 hours; the rate of resection of the small intestine in patients presenting within 12 hours was $14.3 \%$, but it increased to $50 \%$ in patients presenting after 12 hours or more $(P=$ $0.0392)$. Kulah et al. reported that the resection rate in the bowel operated on within 24 hours was $7 \%$, but when the time increased to 48 hours or more, the resection rate increased up to $27 \%$ [14]. The results of this study did not agree with Ohira et al's results. LDH was elevated in both incarceration and strangulation groups but it was failed to predict necrosis.

In this study we aimed to predict intestinal necrosis by IMA levels. It is important to predict necrosis, because incarcerated hernias are more frequently seen in elderly patients, and their prevalence in the over-60-year-old population (added comorbidities and anesthetic risks) has been reported to be $9.8 \%$ compared with $1.8 \%$ for younger patients [26]. Besides it is known that general and spinal anesthesia are both associated with higher rates of serious postoperative complications [27]. The prediction of necrosis can give both the surgeon and the patient the chance of local anesthesia.

Unfortunately the disadvantage of this study is the lack of evaluating other predictive values like CPK levels and since this study is an animal study, pospective human studies must be performed using IMA to be able to suggest that IMA is a gold standard to predict strangulation in the hernia sac.

\section{Conclusion}

IMA is a new marker of ischemia and its place in general surgery is not well known yet. But this study gives cause to hope its usage in incarcerated/strangulated hernias. Further studies should be done to predict intestinal necrosis in the hernia sac, to be able to protect old and unfit patients from the anesthesia associated mortality and morbidities.

\section{References}

[1] D. Roy, J. Quiles, G. Aldama et al., "Ischemia modified albumin for the assessment of patients presenting to the emergency department with acute chest pain but normal or non-diagnostic 12-lead electrocardiograms and negative cardiac troponin T," International Journal of Cardiology, vol. 97, no. 2, pp. 297-301, 2004.

[2] S. Turedi, A. Gunduz, A. Mentese et al., "Value of ischemiamodified albumin in the diagnosis of pulmonary embolism," The American Journal of Emergency Medicine, vol. 25, no. 7, pp. 770-773, 2007.

[3] H. B. Devlin, Management of Abdominal Hernias, Butterworths, London, UK, 1988.

[4] M. A. Smith and N. J. Soper, "Small intestine," in The Washington Manual of Surgery, G. M. Doherty, J. B. Meko, J. A. Olson et al., Eds., pp. 216-227, JB Lippincott, Philadelphia, Pa, USA, 2nd edition, 1999.

[5] N. J. Andrews, "Presentation and outcome of strangulated external hernia in a district general hospital," British Journal of Surgery, vol. 68, no. 5, pp. 329-332, 1981. 
[6] D. Bar-Or, E. Lau, and J. V. Winkler, "A novel assay for cobaltalbumin binding and its potential as a marker for myocardial ischemia-a preliminary report," Journal of Emergency Medicine, vol. 19, no. 4, pp. 311-315, 2000.

[7] D.-H. Lee, H.-K. Jeon, H.-J. Park et al., "Change in ischemiamodified albumin and its clinical significance during exercise stress testing," Circulation Journal, vol. 74, no. 3, pp. 484-489, 2010.

[8] E. Sbarouni, P. Georgiadou, and V. Voudris, "Ischemia modified albumin changes-review and clinical implications," Clinical Chemistry and Laboratory Medicine, vol. 49, no. 2, pp. 177-184, 2011.

[9] A. Wudkowska, J. Goch, and A. Goch, "Ischemia-modified albumin in differential diagnosis of acute coronary syndrome without ST elevation and unstable angina pectoris," Kardiologia Polska, vol. 68, no. 4, pp. 431-437, 2010.

[10] N. V. Bhagavan, E. M. Lai, P. A. Rios et al., "Evaluation of human serum albumin cobalt binding assay for the assessment of myocardial ischemia and myocardial infarction," Clinical Chemistry, vol. 49, no. 4, pp. 581-585, 2003.

[11] A. Gunduz, S. Turkmen, S. Turedi et al., "Time-dependent variations in ischemia-modified albumin levels in mesenteric ischemia," Academic Emergency Medicine, vol. 16, no. 6, pp. 539543, 2009.

[12] J.-P. Laussac and B. Sarkar, "Characterization of the copper(II)and nickel(II)-transport site of human serum albumin. Studies of copper(II) and nickel(II) binding to peptide 1-24 of human serum albumin by 13C and 1H NMR spectroscopy," Biochemistry, vol. 23, no. 12, pp. 2831-2838, 1984.

[13] E. Mcgugan, H. Burton, S. J. Nixon, and A. M. Thompson, "Deaths following hernia surgery: room for improvement," Journal of the Royal College of Surgeons of Edinburgh, vol. 45, no. 3, pp. 183-186, 2000.

[14] B. Kulah, A. Polat Duzgun, M. Moran, I. H. Kulacoglu, M. Mahir Ozmen, and M. S. F. Coskun, "Emergency hernia repairs in elderly patients," The American Journal of Surgery, vol. 182, no. 5, pp. 455-459, 2001.

[15] B. Kulah, I. H. Kulacoglu, M. T. Oruc et al., "Presentation and outcome of incarcerated external hernias in adults," The American Journal of Surgery, vol. 181, no. 2, pp. 101-104, 2001.

[16] B.-J. Ge, Q. Huang, L.-M. Liu, H.-P. Bian, and Y.-Z. Fan, "Risk factors for bowel resection and outcome in patients with incarcerated groin hernias," Hernia, vol. 14, no. 3, pp. 259-264, 2010.

[17] H. Nilsson, G. Stylianidis, M. Haapamäki, E. Nilsson, and P. Nordin, "Mortality after groin hernia surgery," Annals of Surgery, vol. 245, no. 4, pp. 656-660, 2007.

[18] N. A. Bickell, A. H. Aufses Jr., M. Rojas, and C. Bodian, "How time affects the risk of rupture in appendicitis," Journal of the American College of Surgeons, vol. 202, no. 3, pp. 401-406, 2006.

[19] H. Derici, H. R. Unalp, A. D. Bozdag, O. Nazli, T. Tansug, and E. Kamer, "Factors affecting morbidity and mortality in incarcerated abdominal wall hernias," Hernia, vol. 11, no. 4, pp. 341-346, 2007.

[20] G. P. McEntee, A. O'Carroll, B. Mooney, T. J. Egan, and P. V. Delaney, "Timing of strangulation in adult hernias," British Journal of Surgery, vol. 76, no. 7, pp. 725-726, 1989.

[21] G. Ohana, I. Manevwitch, R. Weil et al., "Inguinal hernia: challenging the traditional indication for surgery in asymptomatic patients," Hernia, vol. 8, no. 2, pp. 117-120, 2004.
[22] W. Silen, M. F. Hein, and L. Goldman, "Strangulation obstruction of the small intestine," Archives of Surgery, vol. 85, pp. 121129, 1962.

[23] T. Maeda, H. Yuasa, T. Arimura, S. Endou, K. Akashi, and K. Ashikawa, "Value of the level of serum creatine phosphokinase for early diagnosis of strangulation ileus," The Japanese Journal of Gastroenterological Surgery, vol. 26, pp. 1007-1012, 1993.

[24] S. Ohira, H. Hasegawa, S. Ogiso et al., "Clinical study of the strangulated Ileus without previous abdominal operation," The Japanese Society for Emergency Medicine, vol. 21, pp. 687-693, 2001.

[25] J. A. Álvarez, R. F. Baldonedo, I. G. Bear, J. A. S. Solís, P. Álvarez, and J. I. Jorge, "Incarcerated groin hernias in adults: presentation and outcome," Hernia, vol. 8, no. 2, pp. 121-126, 2004.

[26] D. C. Lewis, C. G. Moran, and K. D. Vellacott, "Inguinal hernia repair in the elderly," Journal of the Royal College of Surgeons of Edinburgh, vol. 34, no. 2, pp. 101-103, 1989.

[27] D. V. Young, "Comparison of local, spinal, and general anesthesia for inguinal herniorrhaphy," The American Journal of Surgery, vol. 153, no. 6, pp. 560-563, 1987. 


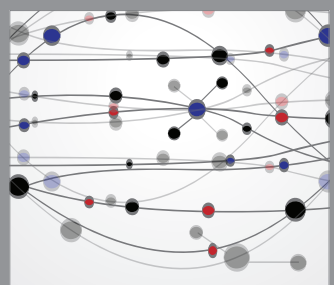

The Scientific World Journal
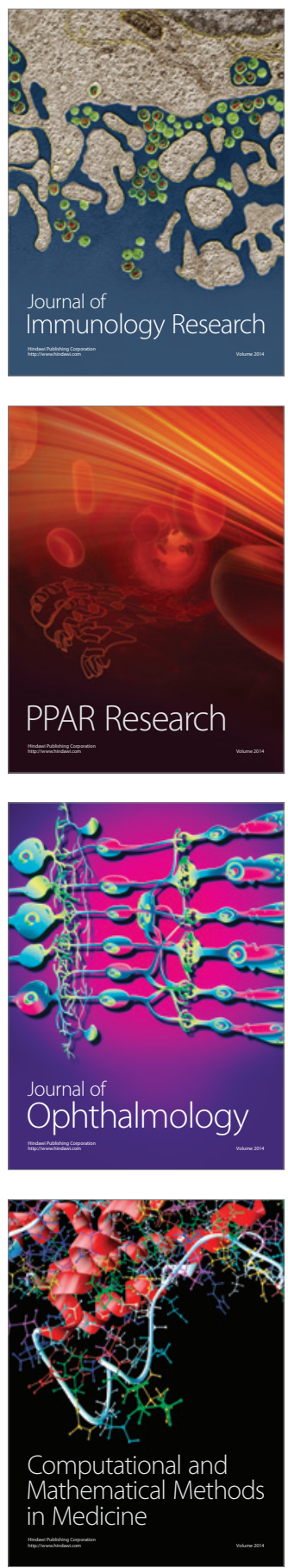

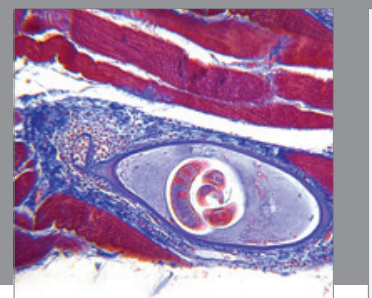

Gastroenterology

Research and Practice
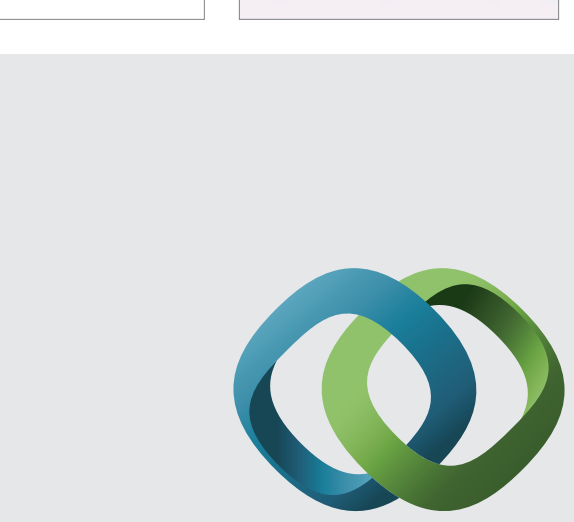

\section{Hindawi}

Submit your manuscripts at

http://www.hindawi.com
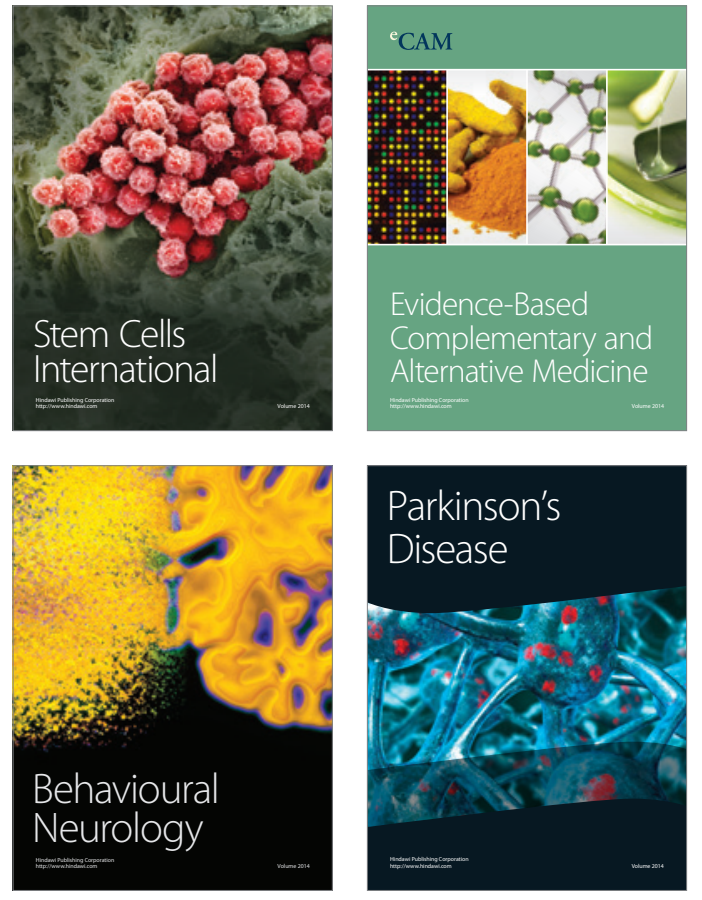
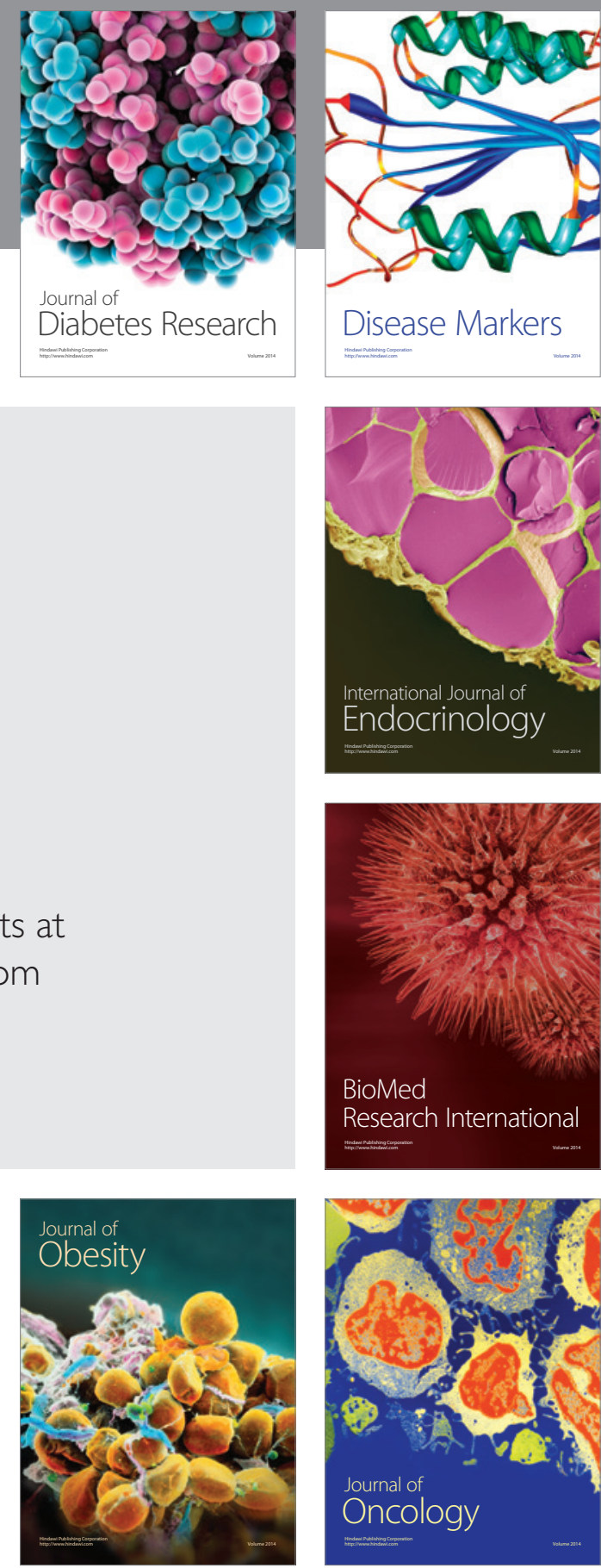

Disease Markers
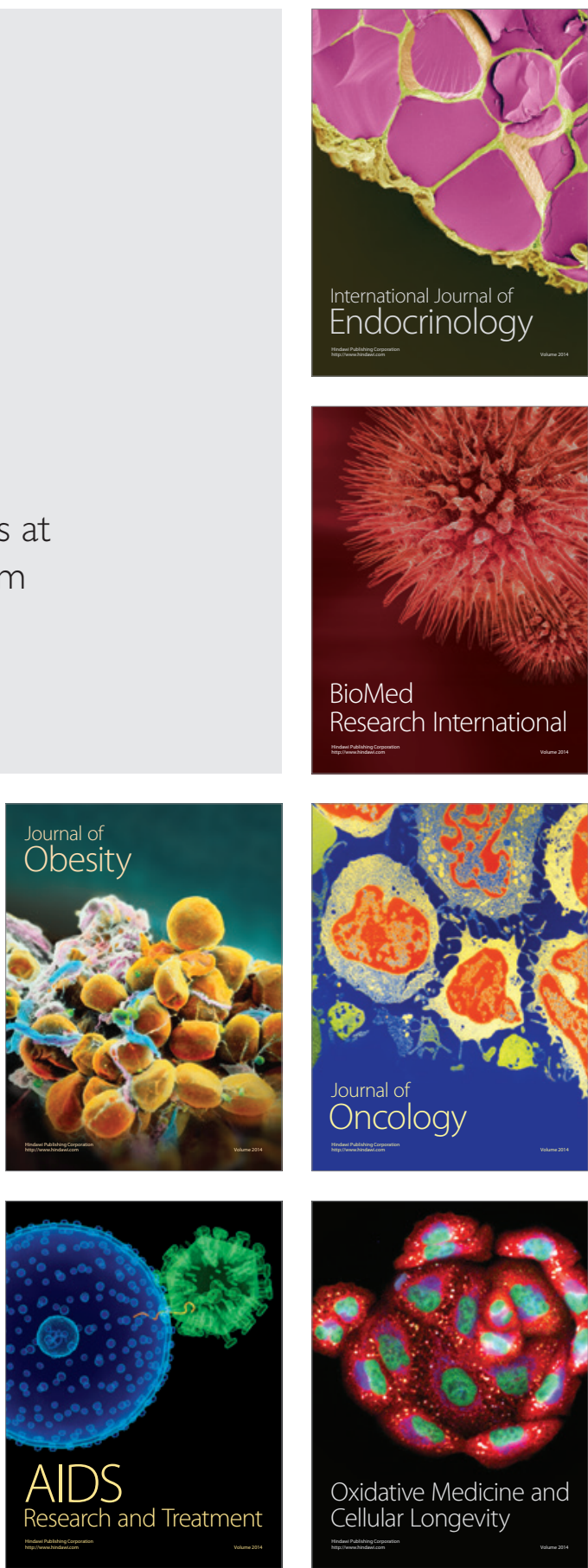